

\title{
Metastable States, Relaxation Times and Free-energy Barriers in Finite Dimensional Glassy Systems
}

\author{
Silvio Franz \\ The Abdus Salam International Centre for Theoretical Physics, \\ Strada Costiera 11, P.O. Box 586, I-34100 Trieste, Italy
}

\begin{abstract}
In this note we discuss metastability in a long-but-finite range disordered model for the glass transition. We show that relaxation is dominated by configuration belonging to metastable states and associate an in principle computable free-energy barrier to the equilibrium relaxation time. Adam-Gibbs-like relaxation times appear naturally in this approach.
\end{abstract}

\section{INTRODUCTION}

Metastable states and configurational entropy, are central concepts in our understanding of low temperature relaxation of disordered and glassy systems [1]. In presence of time scale separation between "fast" and "slow" degrees of freedom relaxation is often described as a walk between metastable states, intended as regions of configuration space where the fast degrees of freedom reach a state of quasi equilibrium before the relaxation of slow degrees of freedom can effectively take place. According to the Adam and Gibbs theory [2] the configurational entropy, i.e. the logarithm of the multiplicity of metastable states is related to the relaxation time by an inverse proportionality relation.

The relation between metastability and relaxation is well understood in infinite range spin glasses with "random first order transition" [3]. The dynamics of these systems is exactly described by the equations of Ideal Mode Coupling Theory [4]. The Mode Coupling transition, spurious in liquid systems, is here driven by the existence of metastable states capable to trap the system for time scales diverging with the systems size. The divergence is of course a Mean Field artifact, but features of mean-field metastable states could well have a reflex in finite dimensional glassy systems [5].

A well known problem in the study of metastable states is that while they are easy to define within mean field theory, their definition becomes elusive in finite dimensions. This problem has been studied in large detail in the context of systems with first order phase transition, where the problem of metastability, can be considered as satisfactorily understood [6,7]. Unfortunately, this is not the case in glassy systems, where despite the appeal of a description of dynamics in terms of metastable states, only very rare contributions have tried to clarify the meaning of metastability beyond the phenomenological level [8].

The "random first order transition scenario" has led to phenomenological attempts to treat the problem of glassy relaxation, and ergodicity recovery in analogy with kinetics of ordinary first order phase transition [9-11]. Liquids at low temperature would appear as "mosaic states", in which locally frozen variables' configurations could be composed combinatorially on a large scale. The typical size of the rigid regions could be computed in a nucleation theory, with the configurational entropy playing the role of an ergodicity restoration bulk driving force, competing with some postulated interface tension. These developments stimulated first principle calculations in microscopic disordered models. In ref. [12] and later in [13] a statistical description of the ergodic state and a computation of the glassy coherent length below $T_{c}$ was proposed through the asymptotic analysis of a Landau-like glassy effective free-energy derived from microscopic models. Unfortunately, in that papers it was not possible to make an explicit connection between the computed free-energy barrier and the relaxation time of the system. Scope of this letter is to discuss this connection. In order to do that, we start from an analysis of glassy relaxation based on separation of time scales. We argue that finite dimensional relaxation is dominated by metastable states that can be characterized along the classical lines of Lebowitz and Penrose (LP) [14], first proposed to describe metastable phases of matter in presence of first order phase transitions. According to LP, metastable states can be considered as constrained equilibrium ensembles with: 1) an order parameter is homogeneous on a suitable mesoscopic length scale 2) a large time life and 3 ) a very small probability of return once abandoned. In analogy with the work of LP, we use in our analysis models with long-but-finite range Kac kind of interactions, which in our case have a disordered character. These offer the possibility of studying finite dimensional effects in an expansion around mean field, and the local mean-field character of correlations, postulated in the phenomenological mosaic description, appears as a consequence of the range of interaction [15]. 


\section{THE MODEL}

Let us consider a spherical Kac p-spin glass model [12] defined for real spins $\sigma_{i}$ on the $d$-dimensional hypercubic lattice $\Lambda$ of linear size $L$ and Hamiltonian

$$
H(\sigma, J)=-\sum_{i_{1}, \cdots, i_{p} \in \Lambda} J_{i_{1} \cdots i_{p}} \sigma_{i_{1}} \cdots \sigma_{i_{p}}
$$

where the couplings $J_{i_{1} \cdots i_{p}}$ are i.i.d. Gaussian variables with zero average and variance

$$
E\left(J_{i_{1} \cdots i_{p}}^{2}\right)=\frac{1}{2} \gamma^{p d} \sum_{k \in \Lambda} \psi\left(\gamma\left|i_{1}-k\right|\right) \cdots \psi\left(\gamma\left|i_{p}-k\right|\right)
$$

where $p$ is an integer $p \geq 3$ and $\psi(|x|)$, is a non-negative integrable function verifying the normalization $\int d^{d} x \psi(|x|)=$ 1 . With this choice, the couplings $J_{i_{1} \cdots i_{p}}$ are sensibly different from zero only if all pairs of variables $\left|i_{l}-i_{m}\right|_{\sim}^{<} \gamma^{-1}$ $\forall l \neq m=1, \ldots, p$, so that only variables that are at distances $\mid i-j] \stackrel{<}{\sim} \gamma^{-1}$ effectively interact. The effective interaction range $\gamma^{-1}$ will be assumed to be large throughout the paper. We partition the lattice in boxes $B_{x}$ of a coarse graining length size $\ell$ and impose a local spherical constraint $\sum_{i \in B_{x}} \sigma_{i}^{2}=\ell^{d}$ for all $x$. We are interested to the regime where the three defining lengths are supposed, as in LP, to verify the relation

$$
\log L<<\ell<<\gamma^{-1}<<L
$$

and for definiteness we will have in mind the situation where $\ell=\gamma^{-\delta}$ for some $\delta \in(0,1)$. The model is chosen in such a way to reduce to the usual mean-field spherical $p$-spin model in the regime $\gamma L \approx 1$. Let us recall the physics of this case [16] that will be useful in the finite $\gamma$ case. On lowering the temperature from the paramagnetic region one encounters two transitions. There is a first transition at the Mode Coupling temperature $T_{c}$ where ergodicity is broken. Below $T_{c}$ an exponential number of ergodic components $\mathcal{N} \approx e^{N \Sigma(T)}$ dominates the thermodynamics. The configurational entropy $\Sigma(T)$ decreases as a function of the temperature, until it becomes zero at a "Kauzmann temperature" $T_{K}<T_{c}$, where a second transition is met.

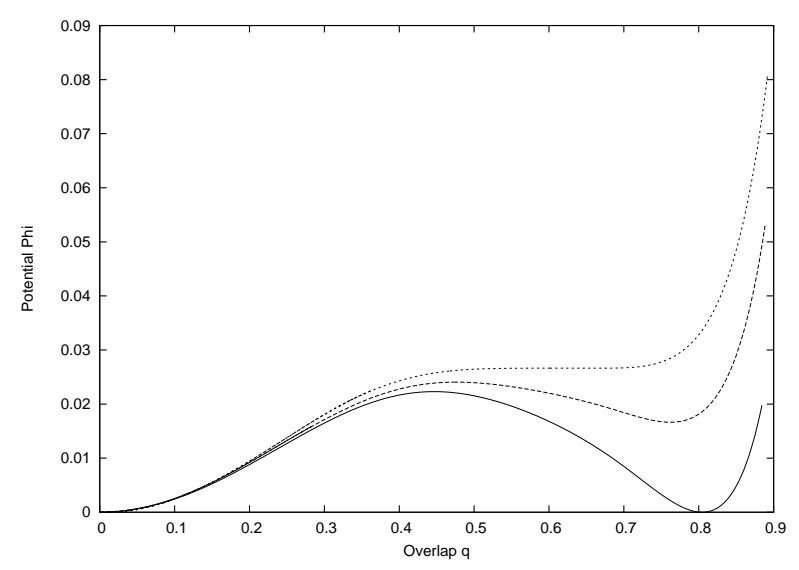

FIG. 1. Qualitative behavior of the mean field potential. From top to bottom the $T=T_{c}, T_{K}<T<T_{c}$ and $T=T_{K}$. The difference of free-energy between the two minima is the product of the configurational entropy ad the temperature.

This structure of states can be studied through the so-called "quenched glassy potential" that considers the freeenergy $\Phi(q)$ of a system $\sigma$ constrained to have a fixed global overlap $q=\frac{1}{L^{d}} \sum_{i} \sigma_{i} \sigma_{i}^{*}$ with a typical reference equilibrium configuration $\sigma^{*}$ [17]. This function has the shape depicted in figure 1, in the region $T_{K}<T<T_{c}$ one finds a two minimum structure analogous to the free-energy as a function of the order parameter for mean-field systems with a first-order phase transition. The value of the overlap in the low minimum and high minima represent respectively the typical overlap among random configurations in different and the same ergodic components. Both minima are associated to the exponential multiplicity of ergodic components, and their difference gives directly the configurational entropy times the temperature $\Phi\left(q_{E A}\right)-\Phi(0)=T \Sigma(T)$. 


\section{DYNAMICS AT FINITE $\gamma$.}

We now address the question of restoration of ergodicity for finite $\gamma$ and $T_{K}<T<T_{c}$. The relaxation of model (1) can be conveniently studied through Langevin dynamics

$$
\frac{\partial \sigma_{i}}{\partial t}=\mu_{x}(t) \sigma_{i}-\frac{\partial H}{\partial \sigma_{i}}+\eta_{i}
$$

where $\mu_{x}(t)$ are Lagrange multipliers that enforce in average the local spherical constraint at each time and the $\eta_{i}$ are white noise variables with correlations $E\left(\eta_{i}(t) \eta_{j}(s)\right)=2 T \delta_{i j} \delta(t-s)$. An insight on the dynamics in the regime (3) can be obtained studying the Kac limit $\gamma \rightarrow 0$ after the thermodynamic limit. Through standard dynamical techniques -Martin-Siggia-Rose formalism [18] or dynamical cavity method [19]-, it is possible to conclude [20] that the finite time local equilibrium spin-spin correlation function $C_{x}^{(\gamma)}(t)=\frac{1}{\ell^{d}} \sum_{i \in B_{x}} \sigma_{i}(t) \sigma_{i}(0)$ on the scale $\ell$, tends to a space homogeneous function, $C(t)$, that for temperatures larger then the Kauzmann temperature $T_{K}$ verifies the usual equilibrium mean field equation for the spherical p-spin model [16]

$$
\frac{d C(t)}{d t}=-T C(t)+\frac{p \beta}{2} \int_{0}^{t} d s C^{p-1}(t-s) \frac{d C(s)}{d s} .
$$

This equation predicts ergodicity breaking below $T_{c}$. The equilibrium correlation function in this regime approaches exponentially fast on a scale $\tau_{M F}$ a non zero limit $\lim _{t \rightarrow \infty} C(t)=q_{E A}$. Conversely, for any finite $\gamma>0$, for finite or infinite $L$, the system should be able to escape from the metastable states and $\lim _{t \rightarrow \infty} \lim _{L \rightarrow \infty} C_{x}^{(L, \gamma)}(t)$ coincide with the equilibrium correlation function $\frac{1}{l^{d}} \sum_{i \in B_{x}}\left\langle\sigma_{i}\right\rangle^{2}=0$. In the regime (3), the behavior of $C_{x}^{(\gamma)}(t)$ can be schematically depicted as in figure 2. As a function of time, the small $\gamma$ correlation function remains close to the mean field curve up to some characteristic time $\tau^{(\gamma)}$ diverging when $\gamma^{-1} \rightarrow \infty$ and it decays to zero on longer scales. Since for small enough $\gamma$ this characteristic time is arbitrary larger than the mean field relaxation time $\tau_{M F}$, one finds that the system equilibrates in the region of phase space where the local overlaps with the initial condition are close to $q_{E A}$ before relaxing further. Moreover, one can expect that on time scales of the order of $\tau_{M F}$, the system has homogeneous correlations on the scale of the box size $\ell$, with typical fluctuation from cell to cell of $C_{x}^{\gamma}(t)-C(t)$ scaling as $1 / \ell^{d / 2}$. In turn, assuming independence of the fluctuations beyond some finite length, using extreme statistics one can estimate the maximum deviation $\max _{x}\left[C_{x}^{\gamma}(t)-C(t)\right]$ to be at most of order $\sqrt{\frac{\log L}{\ell^{d}}}$. This, according to our hypothesis, can be made arbitrarily small. The previously discussed properties are enough to characterize the regions explored on scales smaller then $\tau^{(\gamma)}$ as metastable states in the Lebowitz-Penrose sense.

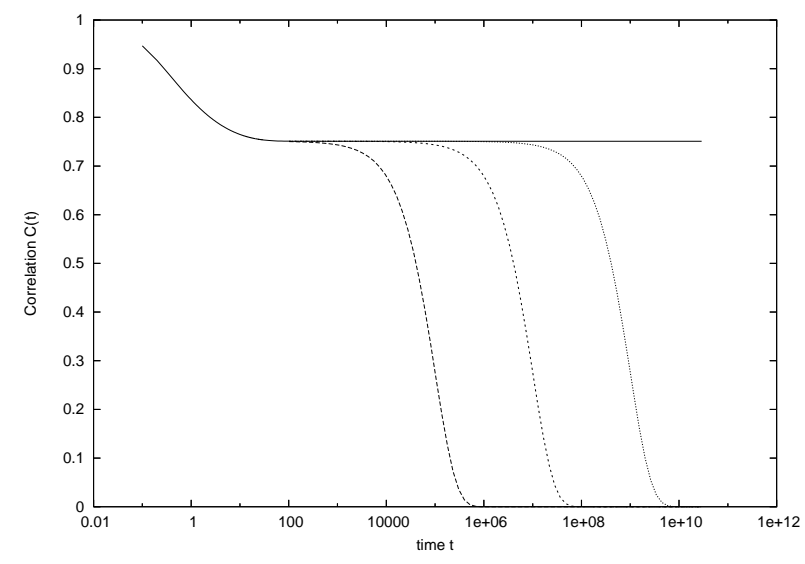

FIG. 2. Qualitative behavior of the correlation function as a function of time for different values of $\gamma$.

The role of the "order parameter", homogeneous in space, is played by the overlap of the configuration of the system with the initial condition $\sigma(0)$. Formally, for any typical equilibrium configuration $\sigma^{*}$, we can define metastable states, as equilibrium ensembles where the configurations $\sigma$ are such that in each box $B_{x}$ the overlap with the initial configuration $q_{x}\left(\sigma, \sigma^{*}\right)=\frac{1}{\ell^{d}} \sum_{x \in B_{x}} \sigma_{i} \sigma_{i}^{*}$ is suitably close to $q_{E A}$. More precisely, one defines an interval $I=\left[q_{-}, 1\right]$, 
with $q_{-}<q_{E A}$ and the set $\mathcal{R}=\left\{\sigma \mid q_{x}\left(\sigma, \sigma^{*}\right) \in I\right\}$. The metastable state is the restricted ensemble

$$
\mu_{\mathcal{R}}(\sigma)=\frac{1}{Z_{\mathcal{R}}} \exp (-\beta H(\sigma)) 1_{\mathcal{R}}(\sigma) .
$$

As in the LP case, the definition of metastable state makes sense for a whole range of values of $q_{-}$, such that a local fluctuation of the overlap larger then $q_{-}$is with high probability pushed back to $q_{E A}$. However, in order for the definition to be useful, the value of $q_{-}$should be fixed a posteriori in such a way that a fluctuation larger then that size should be amplified, and eventually run the system out of the metastable state with finite probability. We can now use this analysis to estimate the relaxation rate of our system as a decay rate of the metastable state. We can then proceeds as in LP, and argue that for the local Langevin dynamics we are using, the relaxation rate $\lambda^{(\gamma)}=\frac{1}{\tau^{(\gamma)}}$ is proportional to $\lambda^{(\gamma)} \approx \frac{Z_{\partial \mathcal{R}}}{Z_{\mathcal{R}}}$ where $\partial \mathcal{R}$ is the set of configuration $\sigma$ belonging to $\mathcal{R}$, such that $q_{x_{0}}\left(\sigma, \sigma^{*}\right)=q_{-}$for at least one point $x_{0} . \lambda^{(\gamma)}$ is in principle a random variable that depends on the initial configuration $\sigma^{*}$ and on the quenched random couplings $J_{i_{1}, \ldots, i_{p}}$. However the relaxation rate should be self-averaging with respect to both sources of noise, and its typical value can be computed considering the average of its log

$$
\log \lambda_{t y p}^{(\gamma)}=E\left(\log Z_{\partial \mathcal{R}}-\log Z_{\mathcal{R}}\right)
$$

where $E$ stands for the canonical average over $\sigma^{*}$ and the quenched average over the distribution of the disorder $J$. Eq. (6) is the main result of this paper. It connects the relaxation rate to a free-energy barrier computable in principle in purely static terms.

\section{RELATION WITH THE EFFECTIVE POTENTIAL.}

For fixed disorder and given finite volume we can define an "effective potential" as the free-energy cost to keep the overlap with an equilibrium configuration $\sigma^{*}$ in each of the boxes $B_{x}$ fixed to specified values $q_{x}$ :

$$
\exp \left(-\frac{\beta}{\gamma^{d}} W_{\sigma^{*}, J}\left[q_{x}\right]\right)=\sum_{\sigma} \prod_{x} \delta\left(q_{x}\left(\sigma, \sigma^{*}\right)-q_{x}\right) e^{-\beta H(\sigma, J)}
$$

where in the expression, we have anticipated the scaling of the free-energy with the interaction range $\gamma^{-1}[21]$. Is is clear how $Z_{\partial \mathcal{R}}$ and $Z_{\mathcal{R}}$ can be related to $W_{\sigma^{*}, J}\left[q_{x}\right]$, in fact, for $\mathcal{S}=\mathcal{R}, \partial \mathcal{R}$, with transparent notation

$$
Z_{\mathcal{S}}=\int_{\mathcal{S}} \mathcal{D} q_{x} e^{-\frac{\beta}{\gamma^{d}} W_{\sigma^{*}, J}\left[q_{x}\right]}
$$

By the previous discussion, the dominant profile in $Z_{\mathcal{R}}$ is estimated as the constant $q_{x}=q_{E A}$ for all $x$. For this profile, the extensive part of $W_{\sigma^{*}, J}\left[q_{x}=q_{E A}\right]$ is a self-averaging quantity: $V\left[q_{E A}\right] \approx E\left(W_{\sigma^{*}, J}\left[q_{x}=q_{E A}\right]\right)$.

For generic overlap profiles $q_{x}$, we can consider $V\left[q_{x}\right]=E\left(W_{\sigma^{*}, J}\left[q_{x}\right]\right)$, and ask if the knowledge of this quantity is enough to reconstruct the relaxation rate $\lambda_{t y p}^{(\gamma)}$ for small $\gamma$.

The restricted partition function $Z_{\partial \mathcal{R}}$ is:

$$
Z_{\partial \mathcal{R}}=\sum_{x_{0}} \int_{\left\{q_{x_{0}}=q_{-} ; q_{x} \in I \forall x\right\}} \mathcal{D} q_{x} e^{-\frac{\beta}{\gamma^{d}} W_{\sigma^{*}, J}\left[q_{x}\right]} .
$$

Each term of the sum on $x_{0}$ will be dominated by a single profile, minimizing $W_{\sigma^{*}, J}\left[q_{x}\right]$ in presence of the constraints. These will be profiles with a localized excitation around $x_{0}$, corresponding to a finite free-energy cost with respect to the uniform state $q_{x}=q_{E A}$.

For fixed $\sigma^{*}$ and $J$, different values of $x_{0}$ could give rise to different free-energy cost and different profile shapes. However, we could argue again that on the mesoscopic scale $\gamma^{-1}$ almost all sites $x_{0}$ would give the contributions of the same order and these dominate the relaxation rate. This is a stronger hypothesis than the other ones underlying our reasoning. It implies that the incipient spatial heterogeneities on a scale $\gamma^{-1}$ responsible for relaxation, have equal probability of appearing in any point of space, independently of the reference configuration $\sigma^{*}$ and the realization of the quenched disorder $J$. Without that, the computation of $\lambda_{t y p}^{(\gamma)}$ should start from an evaluation of (6). This is in principle possible and when done could allow the validation or refutation of the hypothesis. 
Previous work $[10,12,13]$ was implicitly based on that strong hypotheses. Within that framework, if we denote by $q_{x}^{*}$ the profile maximizing $V\left[q_{x}\right]$ for $x_{0}=0$, the relaxation rate will be given by

$$
\lambda_{t y p}^{(\gamma)}=\left(\frac{L}{\ell}\right)^{d} e^{-\frac{\beta}{\gamma^{d}}\left(V\left[q_{x}^{*}\right]-V\left[q_{x}=q_{E A}\right]\right)}
$$

and $V\left[q_{x}^{*}\right]$ will provide a Landau free-energy functional that we can use in a nucleation-like theory. It is clear that restricted to profiles constant in space, $q_{x}=q$, for small $\gamma$ we have: $V\left(q_{x}=q\right)=W_{\sigma^{*}, J}\left[q_{x}=q\right] \approx(L \gamma)^{d} \Phi(q)$, where $\Phi$ is the mean-field potential. In order to compute the free-energy barrier within this self-averaging setting, one should consider spherically symmetric solutions with boundary conditions $\lim _{|x| \rightarrow \infty} q_{x}=q_{E A}$. Formulated in this way the problem reduces to the computation of a critical droplet as suggested by phenomenological theories $[9,11]$. According to conventional nucleation theory [6], close to $T_{K}$ where the two minima are close to degeneracy, one can use the "thin wall approximation" and evaluate the interface tension through the 1D instantonic solutions connecting the two minima. This competes with a bulk driving term given by the free-energy difference between the minima, which in our case is the mean-field configurational entropy. This first principle formulation confirms the prediction of phenomenological theories, that the configurational entropy provides the bulk driving force to ergodicity restoration. The actual computation of $V$ for fixed profile can be performed through a modified version of the replica method [17], needed to average over the spin reference configuration $\sigma^{*}$ and the quenched disorder $J$. This has been done for the model $(1,2)$ in ref. [12]. Without entering in the details of the discussion of that paper we just mention that within a specific replica ansatz it is possible to find a finite surface tension is found for $T$ approaching $T_{K}$. This implies a glassy coherent length behaving as $R_{g} \sim \Sigma(T)^{-1}$ and an Adam-Gibbs free energy barrier $\Delta V \sim \frac{1}{\gamma^{d}} \frac{C}{\Sigma^{d-1}}$. This result is supported by a more elaborated ansatz in [13]. As discussed in [12], and in view of possible non self-averaging effects, these solution could in fact just provide approximations to the true barrier. The "right" replica solutions could change the exponent $d-1$ appearing in the relation. However, the general Adam-Gibbs structure of the formula, that only depends on the fact that the bulk ergodicity restoring force is the configurational entropy, should remain unchanged.

\section{CONCLUSIONS}

Summarizing, we have presented an argument that identifies as Lebowitz-Penrose metastable states the dominant configurations of relaxing glassy systems below the Mode Coupling transition. This allows to compute the relaxation time in purely static term through the evaluation of the probability of equilibrium fluctuations. The relation between relaxation time and free-energy barrier is summarized in eq. (6) which is the main result of this letter. We have also discussed how previous results in the literature relate implicitly to a self-averaging hypothesis. If this holds, the relevant fluctuations have the shape of overlap droplets, and the bulk driving force for ergodicity restoration is provided by the configurational entropy. In agreement with phenomenological theories the relation of the relaxation time with the configurational entropy follows a generalized Adam-Gibbs equation. The argument has been presented in the case of disordered spin model, where playing with the interaction range one can have time scales as much separated as wanted and a mesoscopic scale for which correlations are homogeneous on short time scales. On the other hand our argument is actually only based on the hypothesis that time separation is such to allow partial equilibration of the fast degrees of freedom for fixed values of the slow one, and as such it should be generically valid when this condition is respected.

\section{Acknowledgments}

I would like to thank M. Mézard and G.Parisi for discussions. This work was supported in part by the European Community's Human Potential programme under contract "HPRN-CT-2002-00319 STIPCO".

[1] For a review see: C. A. Angell, Science, 267, 1924 (1995)

[2] G. Adam and J. H. Gibbs, J. Chem. Phys. 43139 (1965)

[3] T.R. Kirkpatrick, P.G. Wolynes, Phys. Rev. B 36, 8552 (1987); T. R. Kirkpatrick, D. Thirumalai, P. G. Wolynes, Phys. Rev. A 40 (1989) 1045

[4] A recent review of Mode Coupling Theory can be found in S. Das, Rev. Mod. Phys. 76 (2004) 785 
[5] For discussion of this point see e.g. M. Mezard, Physica A 30625 (2002)

[6] J.S. Langer, Ann. Phys. 41 (1967) 108. Ann. Phys. 54 (1969) 258.

[7] E. Olivieri, M.E. Vares "Large Deviations and Metastability" CUP 2005.

[8] G. Biroli and J. Kurchan Phys. Rev. E 64, 016101 (2001)

[9] P. G. Wolynes, Jour. Res. NIST, 102187 (1997), X. Xia, P. G. Wolynes, Proc. Nat. Acad. Sci. 97, 2990 (2000), X. Xia, P. G. Wolynes, Phys. Rev. Lett 86, 5526 (2001), V. Lubchenko and P. G. Wolynes, J. Chem. Phys. 1199088 (2003)

[10] G. Parisi, "Gauge Theories, Spin Glasses and Real Glasses" Talk presented at the Oskar Klein Centennial Symposium, cond-mat/9411115, Proceedings of the XIV Sitges Conference "Complex Behaviour of Glassy Systems" 10-14 June 1996. Wolrd Scientific, Singapore 1996.

[11] J.-P. Bouchaud and G. Biroli, J Chem Phys. 1217347 (2004)

[12] S. Franz, J. Stat. Mech. P04001 (2005)

[13] M. Dzero, J. Schmalian and P. G. Wolynes, preprint cond-mat/0502011.

[14] J.L. Lebowitz, O. Penrose, J. Stat. Phys. 3211 (1971)

[15] S. Franz and F. L. Toninelli Phys. Rev. Lett. 92030602 (2004), J. Phys. A: Math. Gen. 37 (2004) 7433

[16] A. Barrat "The p-spin spherical spin glass model" preprint cond-mat/9701031, unpublished.

[17] S. Franz, G. Parisi, J. Phys. I (France) 5 (1995) 1401, Phys. Rev. Lett. 79 (1997) 2486, Physica A 261 (1998) 317

[18] L. F. Cugliandolo in "Slow relaxations and nonequilibrium dynamics in condensed matter" Lecture notes, Les Houches, July 2002, Ed. by Jean-Louis Barrat et al.

[19] M. Mézard, G. Parisi and M.A. Virasoro, Spin glass theory and beyond (World Scientific 1987).

[20] S. Franz, unpublished.

[21] S. Franz and F. L. Toninelli J. Stat. Mech. P01008 (2005) 